\title{
Study of Interaction between Shock Absorber Parameters Influence on Performance using Continuous Flow
}

\author{
Bharath $\mathrm{M}^{\mathrm{a}}$, Dr Badari Kantheti ${ }^{\mathrm{b}}$ \\ ${ }^{\mathrm{a}}$ Jain University, ${ }^{\mathrm{b}}$ MSRIT \\ Bengaluru, Karnataka
}

\begin{abstract}
The oleo pneumatic CFD model was developed to study the interaction between various parameters to understand its influence on the performance of undercarriage shock absorber. The study on continuous fluid flow was focused on to understand the influence of orifice geometry and on other structural parameters including chamber geometry fluid parameters and influence due to interactions among them. The study was carried out using Design of Experiments (DOE) for the responses. The results and outcomes are shown in the form of Pareto plots.
\end{abstract}

Keywords: Orifice Plate, Landing Gear, Undercarriage, CFD, Design of Experiments, oleo pneumatic

\section{INTRODUCTION}

The major requirement of an aircraft undercarriage is to absorb the impact energy at the time of landing and also during taxiing (uneven surfaces) without causing any discomfort to passengers and crew. The rebound of the undercarriage after touch down has to be gradual and not instantaneous. This behavior depends on the shock strut orifice, through which the fluid flows from one chamber to other. Investigations for the impact on undercarriage during aircraft touchdown using design parameters were carried out by many researchers using different approaches like statistical methods, analytical approach and experimental techniques ${ }^{[1]}$. Undercarriage Shock strut/absorber is major structural component which plays a very important role in determining the undercarriage performance and transfers landing load to aircraft structure. The slow extension of undercarriage happens due to the controlled flow of fluid from one chamber to other through an orifice, which directly relates to the performance of the undercarriage ${ }^{[2]}$. Researchers have studied the parameters influencing the undercarriage performance which are rate of flow, orifice discharge coefficient, pressure drop across orifice, orifice dimensions, polytropic index, geometric parameters of chambers (length and diameter), air pressure for, internal gas pressure, hydraulic fluid properties, strut stroke length, un-sprung mass, vertical forces acting on the shock strut, sliding friction, static seal friction, etc., ${ }^{[1,3,4,5,6]}$. Some of these parameters are investigated independently by researchers to understand the influence on an oleo pneumatic shock absorber in order to meet the undercarriage performance requirements ${ }^{[4]}$.

From the literature it is found that, no work was performed to understand the influence / impact due to combination of two or more input parameters and their responses (behavior) due to higher number of unknown parameters. Determination of displacement curve of an oleo pneumatic shock absorber is difficult due to the non-linear motion of the shock absorber ${ }^{[2]}$. Institutions / Industries developed their own methodology to determine the performance of undercarriages by assuming few parameters based on continuous flow, that have major impact but the interactions between these parameters were ignored. Based on the certification agencies requirement, all the undercarriages are validated through testing (drop, test, strength test, fatigue test, etc). Undercarriage testing is an expensive process and iterative in nature to arrive at the final design of shock absorber to ensure smooth and slow extension / rebound of landing gear for improved comfort of crew and passengers in the aircraft.

In this paper, an attempt is made to present the interactions between the parameters during continuous fluid flow help us to understanding of undercarriage shock absorber design performance. The present work discusses the Design of experiment (DOE) method used for arriving at the optimum number of simulations required to assess the interactions between the input parameters, which are critical to the performance of shock strut and at the same time maintaining the accuracy required for assessing the influences of different parameters like orifice geometry (orifice inlet angle, orifice outlet angle, orifice thickness, orifice diameter), chamber geometry (diameter of the lower chamber, \& diameter of the upper chamber Refer Figure 1 for the geometry details), gas pressures, mass flow rate of oil through orifice, etc ${ }^{[3]}$. A factorial design was chosen, since the numbers of parameters to be evaluated are large. The results and outcomes are presented in the form of Pareto charts and the influence on the resultant due to the input parameters along with their interactions are discussed in detail. 


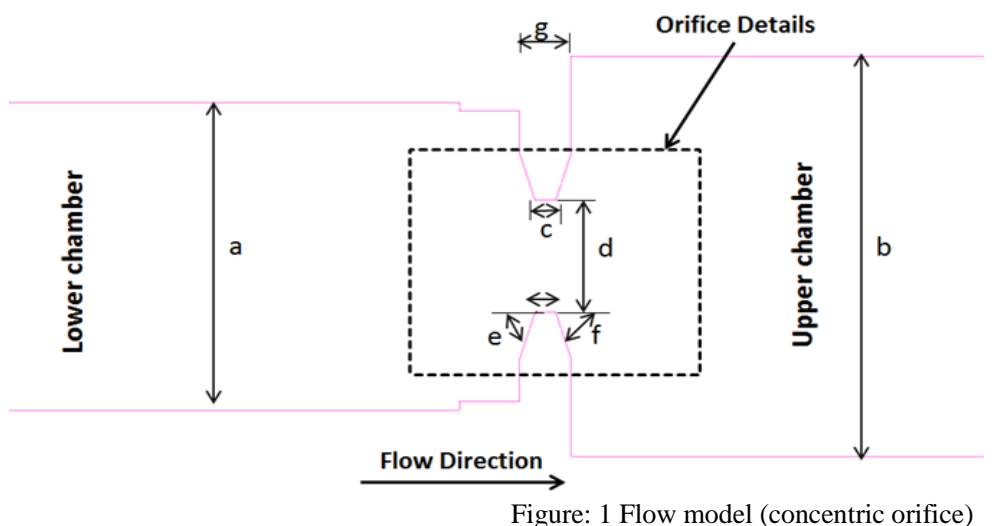

a. Lower chamber diameter

b. Upper chamber diameter

c. Orifice thickness

d. Orifice diameter

e. Orifice inlet angle

f. Orifice outlet angle

g. Orifice base thickness

There are different parameters that needs to be investigated to predict the flow behavior, which can be taken forward for analyzing undercarriage behavior. For example: Reynolds number is important parameter for predicting the type of the flow, i.e. either laminar or turbulent. The study of orifice geometry i.e. the convergent and divergent areas before and after are important, the compression / damping load that is developed by forcing the oil through an orifice. Evaluation of the coefficient of discharge is helpful in predicting the pressure losses across the orifice. Studying the interaction between these parameters will give a better understanding of how to choose the appropriate parameter for further studies.

Several studies exist in the literature for the equation of motion for the undercarriage (considering the UC as two degree of freedom system), so that the various performance parameters could be obtained from this equation.

The equation of motion for the linear undercarriage was derived by Flugge ${ }^{[7]}$ as:

$$
\begin{aligned}
& m_{1} \ddot{x_{1}}+b\left(\dot{x_{1}}-\dot{x_{2}}\right)+k_{1}\left(x_{1}-x_{2}\right)=W_{1} \ldots \ldots \ldots \ldots . . \\
& m_{2} \ddot{x_{2}}-b\left(\dot{x_{1}}-\dot{x_{2}}\right)-k_{1}\left(x_{1}-x_{2}\right)+k_{2} x_{2}=0 .
\end{aligned}
$$

Also the pressure in the upper chamber was defined by Flugge ${ }^{[7]}$.

$$
p_{1}=p_{0}\left(\frac{z_{0} A_{0} / A_{1}}{\frac{z_{0} A_{0}}{A_{1}}-x}\right)^{\gamma}
$$

The values $A_{0}$ and $A_{1}$ indicates the area at different location on the piston. Whereas $\boldsymbol{p}_{\mathbf{0}}$ indicates the pressure in both chambers when strut is fully extended and at rest. $\mathrm{x}$, gamma \& $\mathrm{P} 1$ - explanation

The coefficient of discharge, which is a very important parameter for the characterization, was given by Benjamin ${ }^{[2]}$ :

$$
Q=C_{d} A_{n} \sqrt{\frac{2}{\rho}\left(p_{h}-p_{a}\right)}
$$

Where,

$A_{n}=$ net orifice area $=\left(\boldsymbol{A}_{\mathbf{0}}-\boldsymbol{A}_{\boldsymbol{p}}\right)$,

$Q=$ Volumetric Rate of discharge

Multivariate optimization generally implies two steps: a) evaluation using factorial design to identify signification factors and $b$ ) an estimation of the response function. The parameters Inlet Angle Outlet Angle, Orifice Thickness (in), Orifice Base Thickness (in), Orifice Diameter (in), Q (mass flow rate) (lb/s) \& Outlet Pressure (psi) were studied for individual influences on the responses ${ }^{[3]}$. The possible interactions between the input parameters and each responses will provide additional interactions terms. In this paper factorial design were used to determine the parameters along with the interactions that influences the responses (Pressure at Orifice Inlet, Velocity at Orifice Inlet, Pressure at Orifice Outlet, Velocity at Orifice Outlet, Pressure at Inlet, Velocity at Inlet, Pressure at Outlet, Velocity at Outlet, Co-efficient of discharge $\left(C_{D}\right)$.

\section{GENERAL METHODOLOGY}

A simplified representative undercarriage shock strut continuous flow model (Figure 1) was developed with an orifice in the center to study the effect of parameters and their interactions during the constant flow of fluid from one chamber to other through an orifice. STAR CCM+ was used throughout for the CFD analysis purpose.

The CFD analysis model was developed based on past experiences using the following physics like three dimensional Space, Steady flow, Oil, Segregated flow, Constant Density, Turbulent \& K-Epsilon Turbulence model, refer Figure 1 for the geometry details.

Each turbulent model has strength and weakness and is suited to a particular regime. The K-epsilon turbulence model is selected as it is robust, computationally cheap, offers good convergence and suitable for parametric studies, which is more relevant to the study discussed here. The fluid used in the analysis is SKYDROL and its properties are given below:

- $\quad$ Density: $0.13 \mathrm{lb} / \mathrm{in} 3$
Viscosity: 0.02408 pa-s


Mesh sizes were kept different for zones. Polyhedral mesh element with surface remesher along with prism layer to capture the boundary layer effects was selected.

For surface mesh, the Star CCM+ has two models, i.e. surface remesher and the surface wrapper. The surface remesher improves the overall quality of the existing surface of the volume mesh models. The surface wrapper is typically used in concurrence with the surface remesher. However as the surface wrapper make available the manifold mesh from a complex geometry, it is not used in the model described in this paper.

The polyhedral mesh is selected as it is numerically more stable, less diffusive, and more accurate than an equivalent tetrahedral mesh. Moreover, also contains approximately five times fewer cells than that of a tetrahedral mesh for a given starting surface. The prism layer meshers are generated next to the wall boundaries to improve the flow accuracy for the enhanced calculation of the several flow features such as pressure drop etc. Figures 2 illustrate general structure similar to undercarriage flow structure. The element size is finalized after various iterations (sensitivity study) such that the flow field could be captured as accurate as possible. Also two volumetric controls are created near the orifice to capture the detailed flow information.

The boundaries are created at the inlet and outlet of the domain to specify the boundary conditions. A unique solution is defined by the boundary condition, and the properties which can be included in the boundaries are pressure, velocity etc. In the present model, the mass flow rate is defined at the inlet and the pressure is defined as the outlet boundary condition.

Some of the experiments were analysed in MATLAB and the model is shown in Figure 2. The path in yellow highlights the liquid flow path.

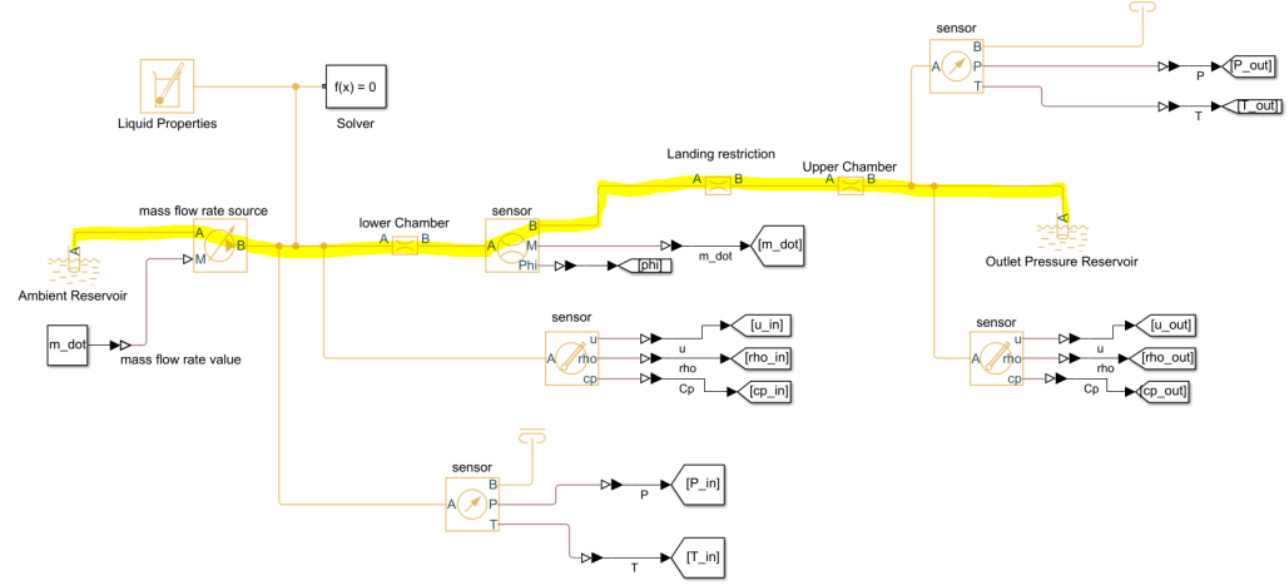

Figure 2: MATLAB fluid flow model

The MATLAB fluid model was carried out without convergent / divergent on orifice parameters and evaluated inlet pressure, outlet velocity. The available model in MATLAB was used and the minimum values in Table - 1 was used for validation.

$m_{A c}=\frac{\mu \mathrm{Re}_{\mathrm{c}} \sqrt{\pi S_{R}}}{2}$

ar $=\frac{S_{\text {or }}}{S_{A}}$ eq 5

$P_{A}-P_{B}=\frac{m_{A}(1-a r) \sqrt{m_{A}+m_{A c}}}{2 c_{d}^{2} S_{R}^{2} \rho_{u}}$ eq 6

Where,

$\dot{m}_{A c}$ is the critical mass flow rate

$\mu$ is the dynamic viscosity

$S_{R}$ is the restriction area

$\mathrm{Re}_{\mathrm{c}}$ is the critical Reynolds number

$S_{A}$ is the nozzle inlet opening

$P_{A}$ is the nozzle inlet pressure

$P_{B}$ is the nozzle outlet pressure

$\dot{m}_{A}$ is the mass flow rate of the system

$c_{d}$ is the coefficient of discharge

$\rho_{u}$ is the upstream densityar is the area ratio

\section{DESIGN OF EXPERIMENT (DOE)}

A design of experiment is a statistical technique for analyzing experiments using series of tests. The factors are the input parameters that are varying independent variables, while the responses are the dependent variables which are measure of the outcome. Factors are the model parameters in which minimum and maximum values of a process or system are considered, so that we may observe the cause and effect relationship between the responses and factors in the process. 


\subsection{DOE Model}

The challenge was to define the DOE methods with minimal number of analysis, to enable DOE modeling with a feasible amount of effort. DOE is chosen to reduce the number of analysis required by ensuring the uniformity and to capture all possible combinations of design points. A conventional and useful form of DOE is the factorial design $2^{\mathrm{k}}$. This is economical while also effectively covering the entire design space. Factorial design is the most widely used method, for evaluating large number of factors in a limited set of experiments. Different fractional factorial designs can be created to estimate the interaction effects separately.

There are seven (7) input parameters considered for this study against nine (9) output responses measuring influence of orifice geometry, chamber geometry, fluid pressure and mass flow rate through the orifice as shown in figure 3 :

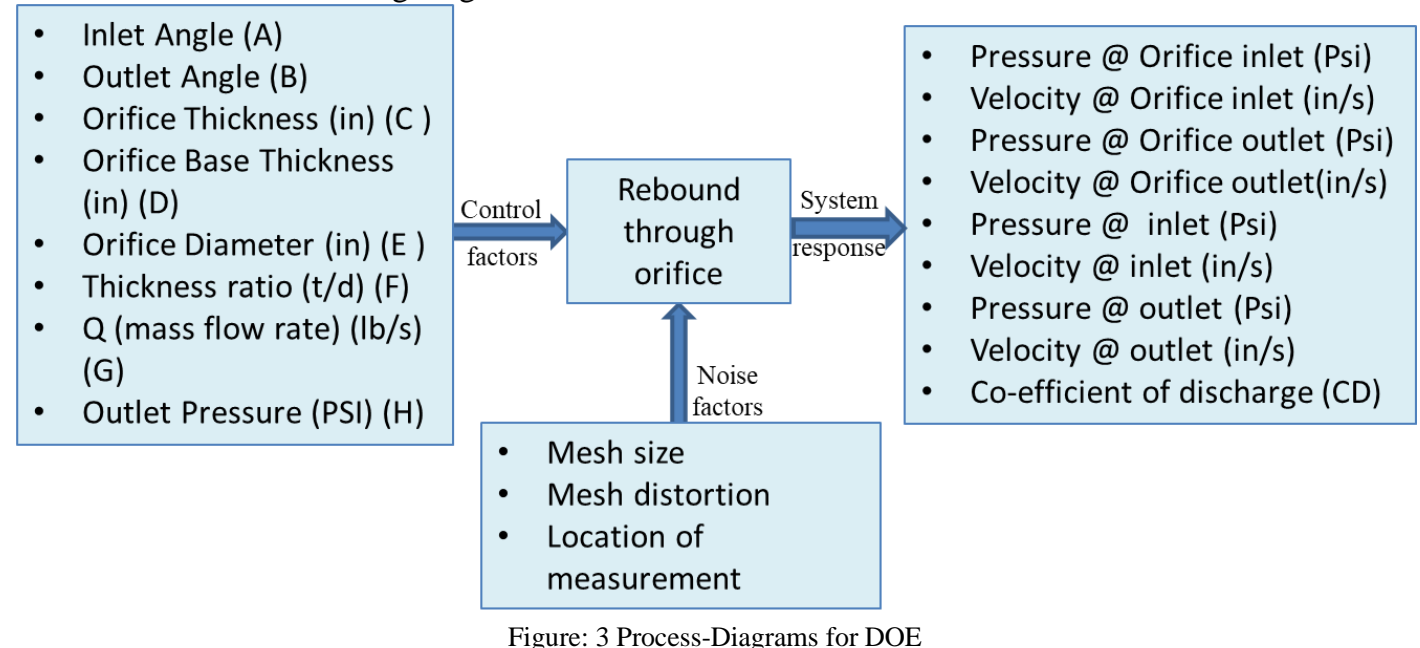

The independent factors (variables) ranging from minimum to maximum values that best meet the design objectives is given below

\begin{tabular}{|l|c|c|}
\hline Parameters & Minimum & Maximum \\
\hline Inlet Angle & 0 & 60 \\
\hline Outlet Angle & 0 & 60 \\
\hline Orifice Thickness (in) & 0 & 0.2 \\
\hline Orifice Base Thickness (in) & 0.3 & 0.8 \\
\hline Orifice Diameter (in) & 0.2 & 1 \\
\hline Q (mass flow rate) (lb/s) & 55 & 165 \\
\hline Outlet Pressure (psi) & 300 & 6000 \\
\hline
\end{tabular}

Table 1: Input parameters

The analyzed factors are a mixture of factors (maximum and minimum) along with mid value, which allows studying the effect of the variation of the ratios among the variables.

\section{Analysis results \& discussions}

The analysis were carried out using CFD software Star $\mathrm{CCM}+$ and the results were studied Pareto charts for various parameters like Pressure and velocity @ Orifice inlet and outlet, pressure and velocity at inlet and outlet of the chamber. Display and processing of the simulation results are more relevant to this study are divided into various categories. The maximum value of average velocity is obtained near the orifice, i.e., where the flow is exiting the orifice, which is quiet obvious phenomenon as the area is diverged near the orifice. Thus the separation of flow can be seen after the orifice exit area.

The flow initiation starts from the inlet and proceeds towards the left of the model. The mass flow rate which is directly related to the velocity of the flow is one of the key parameter to evaluate the effect on different response parameters.
Even the absolute total pressure varies from inlet to outlet. It can be seen that maximum value of the absolute total pressure starts decreasing once the flow crosses through the orifice. The major effect of any parameter (The minimum value to the maximum value) can be observed only near the orifice that means the orifice area is very critical to the various response parameters.

The interpretation of the factorial design DOE were presented using the Pareto chart, from which the effect of individual parameter on the various individual responses can be predicted. The input parameters shown in Figure 3 are used to predict the consequence on the response parameters.

\subsection{Orifice inlet Pressure:}

The Pareto charts (Figures 5) from the DOE results analyzing the effect on various input parameters along with the interaction on two, three parameters using polynomial equation on the orifice inlet pressure on measured responses are represented in the table 2 for various confidence levels. 
Based on the results obtained for pressure at orifice inlet, the response polynomial coefficients were determined in order to evaluate the statistical significance for each coefficient / responses using Pareto charts (Figures 4). All coefficients with a "t value of 1.98 " or above were considered significant.

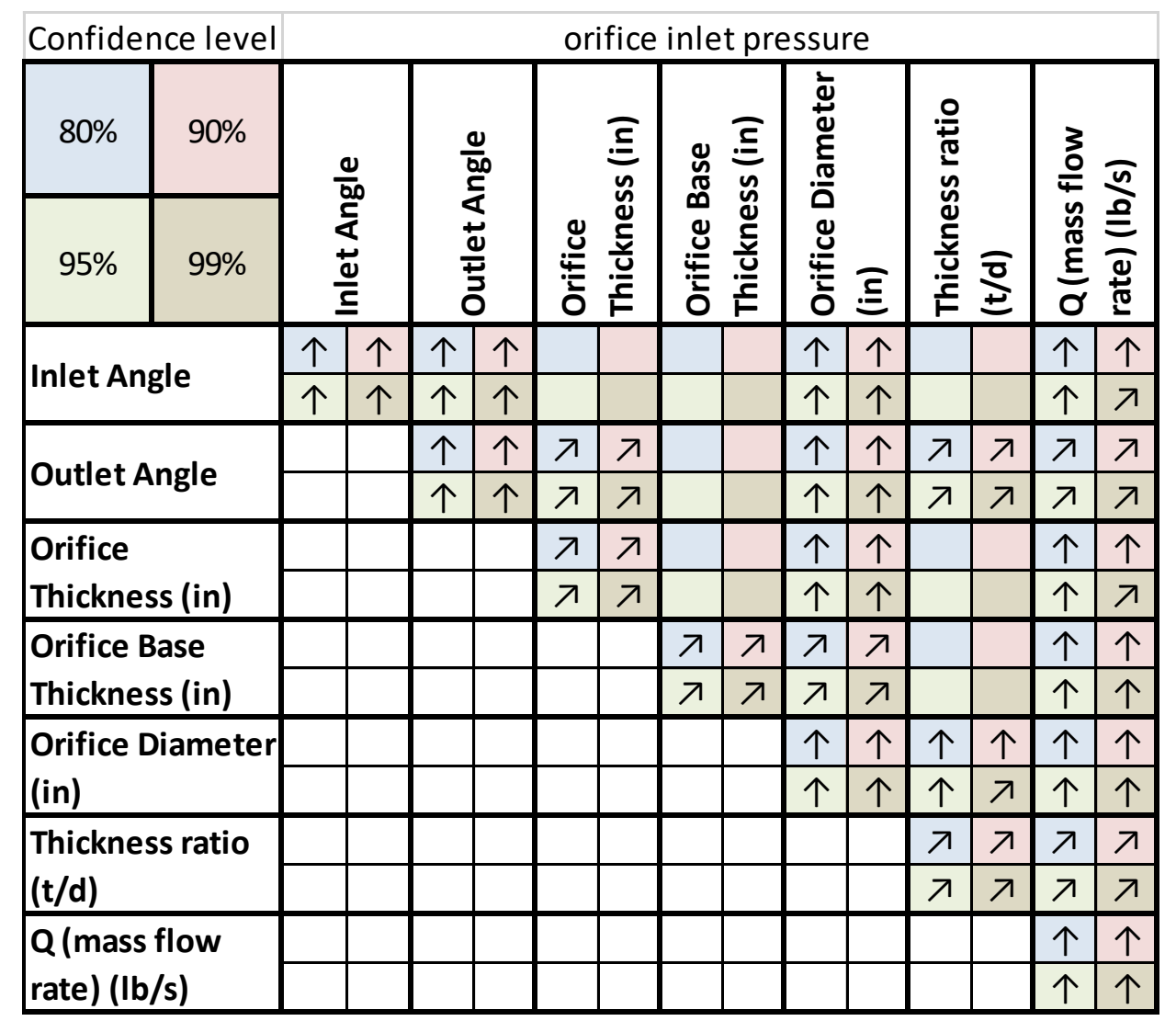

Table 2: Orifice inlet pressure

The above table shows that the orifice area and mass flow rate has a significant effect on the response, and also when they are combined ( 2 factors) with inlet $\&$ outlet angle and thickness of the orifice has significant effect. The inlet and outlet angle of the orifice has moderate effect on the inlet pressure, whereas the interaction amongst them has higher influence on the orifice inlet pressure. As the number of interacting parameter increases from two to three and four the trend remains same except that the orifice thickness along with interactions with other input parameters shows good influence on the orifice inlet pressure.

Orifice inlet velocity, Orifice Outlet pressure \& Orifice Outlet velocity follows similar trends (influence of input parameters) and follows "Bernoulli equation for steady frictionless incompressible flow along a streamline"

$$
\frac{p_{1}}{\rho}+\frac{1}{2} V_{1}^{2}+g z_{1}=\frac{p_{2}}{\rho}+\frac{1}{2} V_{2}^{2}+g z_{2}=\text { const }
$$

The figure 4 shows the influence due to interactions between more input parameters and their influences on Orifice inlet Pressure response. 


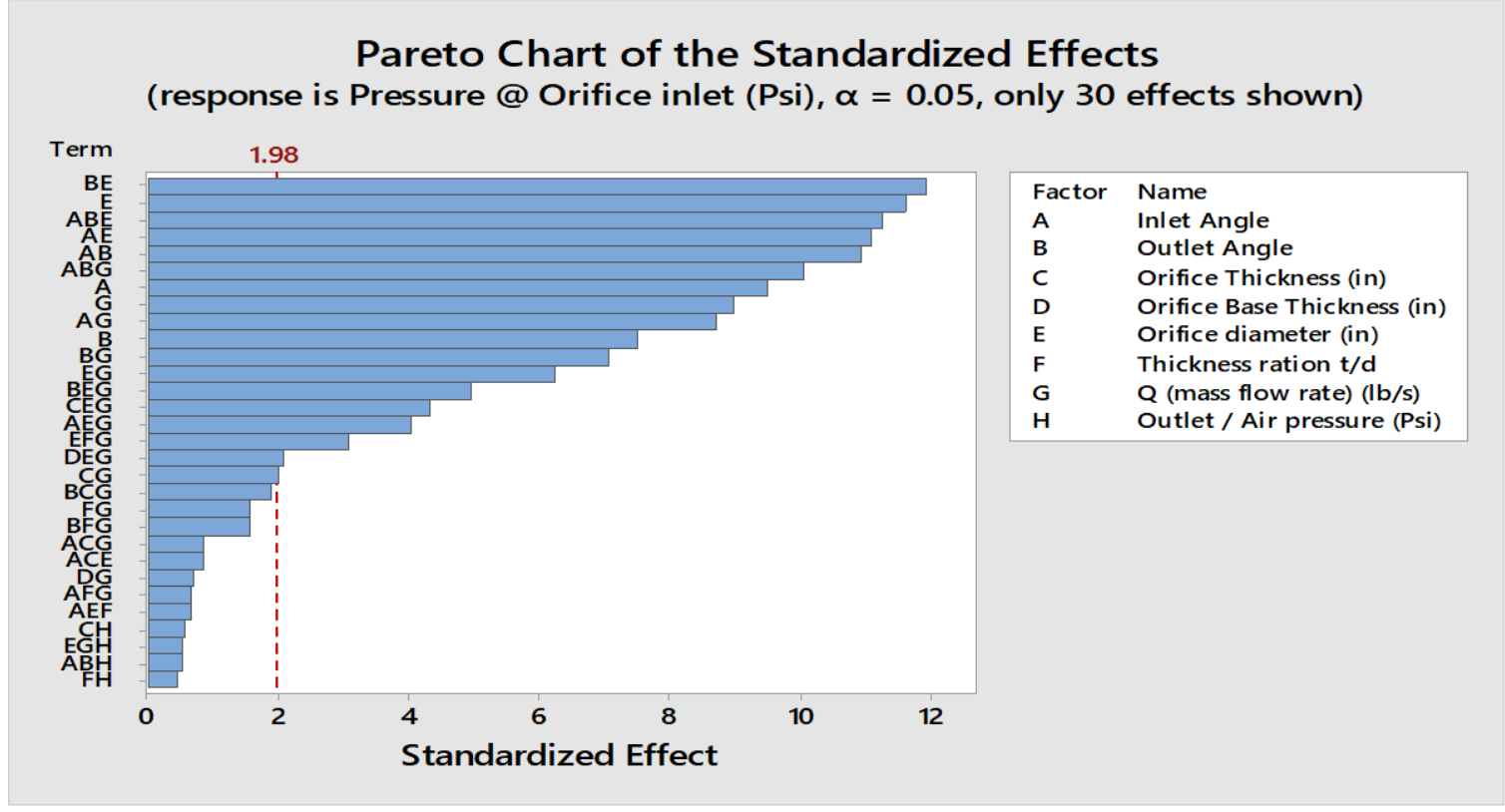

Figure 4: Pareto chart (multiple parameters interactions) for orifice inlet pressure

The non-significant response coefficients were deleted and the following significant polynomial response equations for pressure at orifice inlet were generated. There is a good correlation among the models predicted $\mathrm{SE}$ and the experimental values $\mathrm{R}^{2}=0.8058$

The table shows that the orifice area and mass flow rate and their combinations are combined ( 2 or more factors) with inlet \& outlet angle also have significant effect. The inlet and outlet angle of the orifice has moderate effect on the inlet pressure, whereas the interaction of them has higher influence on the orifice inlet pressure. As the number of interacting parameter increases from two to three (figure 4) and the trend remains same for the velocity at orifice inlet, pressure at orifice outlet, velocity at orifice outlet, pressure at inlet, velocity at inlet, inlet Pressure and outlet pressure

\subsection{Coefficient of discharge $\left(C_{d}\right)$}

The figure 5 shows the influence due to interactions between more input parameters and their influences on coefficient of discharge.

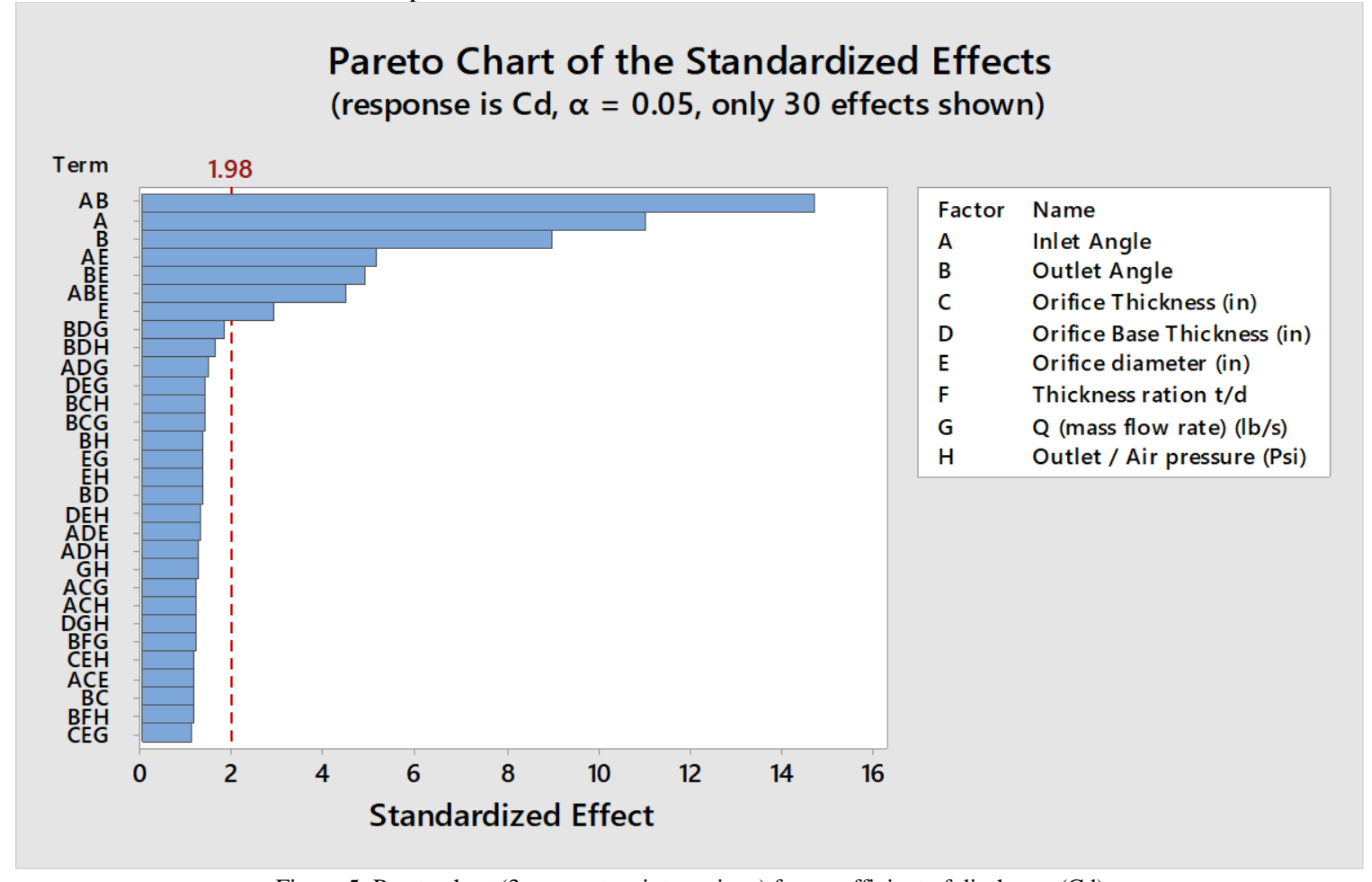

Figure 5: Pareto chart (3 parameters interactions) for co-efficient of discharge (Cd) 
The non-significant response coefficients were deleted and the following significant polynomial response equations for pressure at orifice inlet were generated. There is a good correlation among the models predicted $\mathrm{SE}$ and the experimental values $R^{2}=0.8629$

The above Pareto chart (figure 5) shows that the inlet angle, outlet angle and orifice area has significant effect on the response and has good influence with interacting among them ( 2 way as well as three way interaction).

\section{SUMMARY / CONCLUSIONS}

Shock absorber CFD model was developed to identify the parameters which will influence the behavior of Landing Gear shock absorber using continuous flow and using design of experiments. Even though, these analysis was for the continuous flow, but the number of parameters to be evaluated using constant volume in constrained flow \& multi-phase problem required for shock absorber can be reduced.

The analysis was carried out for 7 different input parameters, with traditional design of experiment to study the overall global trend and identification of key parameters based on the individual and interactions responses for further analysis in a contained volume, constrained flow \& multi-phase problem. The parameters influencing the output variables were primarily found to be Orifice diameter, Mass flow rate, Outlet angle \& Orifice Base Thickness are identified for further evaluation based on sensitivity analysis. As a result of the increasing complexity in CFD analysis, several responses should be simultaneously studied / optimized. Further work involving the study of the output vs key parameters may be taken up in the domain of interest using modern technique such as LATIN HYPER CUBES \& GAUSSIAN SURFACE RESPONSE to ascertain the nonlinear behavior accurately.

\section{REFERENCE}

[1] An Experimental study of orifice Coefficients, Internal strut pressure and loads on a small oleo-pneumatic shock strut. Jamesh H Walls, April 1955.

[2] Analysis of Landing-Gear Behavior. Benjamin Milwitzky and Francis E. Cook, March 1952.

[3] Determination of influence of parameters on Undercarriage shock absorber, Bharath M, Pallavi Singh \& Dr. Kantheti Badari Narayana, SAE International, 2018

[4] Shock absorber modelling. Henrik Skagerstrand, 2014.

[5] Some Theoretical Studies Concerning Oleo Damping Characteristics, H. Hall. 1967.

[6] Discharge Coefficient Performance of Venturi, Standard Concentric Orifice Plate, V-Cone, and Wedge Flow Meters at Small Reynolds Numbers, Colter L. Hollingshead, 2011.

[7] Landing-Gear Impact. W. Flugge, October 1952.

[8] Flow through Pipe Orifices at Low Reynolds Numbers, F. C. JOHANSEN, October 1, 1929. 